\title{
The prevalence of strabismus in schizophrenic patients in Durban, KwaZulu Natal
}

\author{
D Ndlovu, S Nhleko, Y Pillay, T Tsiako, N Yusuf and R Hansraj*
}

Discipline of Optometry, Faculty of Health Sciences, University of Kwazulu Natal, Private Bag X54001, Durban, 4000 South Africa

$<$ hansrajr@ukzn.ac.za>

Received 17 December 2010; revised version accepted 15 August 2011

\section{Abstract}

Purpose: The psychosocial implications of a strabismus have not received sufficient attention and yet may have a significantly negative effect on a person's life. Schizophrenia is the fourth leading cause of mental disability in the developed world. Both schizophrenia and strabismus appear to share a genetic and neurological origin. This study thus set out to assess the prevalence of strabismus in a sample of schizophrenic subjects. Setting: The study was carried out in Durban, Kwazulu-Natal in South Africa specifically at the Durban and Coastal Mental Health Institution. Method: Fifty schizophrenic subjects residing at the Durban and Coastal Mental Health (DMH) Institution were assessed for the presence of strabismus using the cover test and modified Krimsky test. The inclusion criteria were all subjects diagnosed with schizophrenia, of both genders and any race. The subjects were between fifteen to seventy years of age. The exclusion criteria included any other mental illness, systemic disease or medication other than that used on schizophrenic patients. Results: The prevalence of strabismus in this sample was found to be $74 \%$ which was considerably higher than that reported by other studies ${ }^{1,2}$. There was a greater incidence of exotropia as opposed to esotropia. The mean distance exotropia as detected with the cover test was 33 prism dioptres. Similarly, the near cover test indicated a mean of 11 prism dioptres exotropia, and with the modified Krimsky test a mean of 9 prism dioptres exotropia. Most of the strabismic subjects $(86.5 \%)$ presented with a strabismus when fixating a near target. Generally the strabismus was found to be constant and unilateral and no gender bias was found. Conclusion: There appears to be a strong relationship between strabismus and schizophrenia. Health care professionals, especially those dealing with paediatric care, parents and the public should be made aware of this relationship so that psychological advice and early intervention can be sought for strabismic children. (S Afr Optom 2011 70(3) 101-108)

Key words: Schizophrenia, strabismus, cover test, modified Krimsky test, heterotropia, genetics.

\section{Introduction}

A strabismus or heterotropia is a manifest deviation of one or more of the visual axes, commonly referred to as a squint ${ }^{3,4}$. A strabismus can be either congenital or acquired for example due to cataracts. The main etiology of strabismus includes mechanical, paretic, refractive and neurological conditions or may be due to systemic conditions such as multiple sclerosis or diabetes ${ }^{3,5,6}$. Being a manifest deviation, a strabismus has been regarded as a facial abnormality $^{7}$. Previous studies ${ }^{8-11}$ have reported that the presence of a noticeable strabismus has a negative effect on the self image and subsequently on the psychosocial makeup of the individual. Furthermore, Satter- 
field et $a l^{9}$ asserted that the negative influence persists and actually intensifies into adulthood. This assertion has been supported by Tonge et al ${ }^{12}$ and more recently by Durnian et al ${ }^{13}$ who postulated that since the presence of a strabismus can affect an individual's interaction with others it can actually lead to psychiatric disorders. Poor social functioning in the teen years has been associated with the later appearance of schizophrenia ${ }^{14}$. Thus far, schizophrenia appears to be the only serious mental health disorder associated with strabismus ${ }^{1,15}$.

Schizophrenia, literally split mind, is a psychotic disorder characterized by major disturbances in thought, emotion, behaviour and disordered thinking in which ideas are not logically related ${ }^{16,17}$. Schizophrenia includes faulty perception and attention; flat or inappropriate affect (where a patient has virtually no emotional response to a stimulus) and bizarre disturbances in motor activity ${ }^{18}$. The exact cause of schizophrenia is not known but is related to disorders in brain structure and chemistry which can be brought about by metabolic disorders, viruses, trauma or abnormal substances such as lead ${ }^{19,20}$. Schizophrenia is said to run in families, which implicates either genetic or environmental factors ${ }^{19-21}$. Most schizophrenic disorders occur in the second and third decades of life ${ }^{14,17}$. Signs of schizophrenia include hallucinations which are perceptual experiences that feel real to the individual even though there is nothing to perceive and delusions which are beliefs that are not perceptually true but which the individual considers real ${ }^{17,20}$.

The risk of schizophrenia has been linked ${ }^{11-13}$ to deletions on chromosome $22 \mathrm{q}$ which is associated with facial abnormalities including a cleft palate ${ }^{14}$. Previous studies ${ }^{22-24}$ have also established an association between schizophrenia and abnormal eye movements involving pursuits and saccades. There appears to be a link between the neurological etiology of strabismus and schizophrenia, and this link is thought to be genetic in origin ${ }^{1-3}$. Past literature ${ }^{2,25}$ have revealed two genes known as PMX2B and ARIX, which, when defective, has been found to have a strong correlation to both strabismus and schizophrenia. The ARIX gene is known to encode a transcription factor protein $^{25}$ shown to be responsible for the development of cranial nerves III and IV. Although previous studies ${ }^{1,15}$ suggest a link between strabismus and mental disorders, the psychosocial implications of a strabismus have not been given sufficient attention. Furthermore, the prevalence of strabismus in schizophrenic patients has not been widely studied $^{2}$. Such studies are expected to benefit both parents and individuals concerned with paediatric care by raising awareness of the link between strabismus and schizophrenia and by changing the treatment approach to some strabismic subjects to include monitoring behavioural changes, regular psychological assessments and counselling. Following a literature review, only three studies $1,2,15$ have been found specifically investigating the link between strabismus and schizophrenia. One study by Toyota et $a l^{2}$ that established the association between strabismus and schizophrenia was restricted to only Japanese people which may have biased implications when considering the genetic links. The study by Schiffman et al 15 investigated ocular alignment deficits on children whose parents had been diagnosed with schizophrenia. A more recent study by Mohney et al limited the sample to subjects younger than 19 years of age, and was not restricted to schizophrenia. It has been found, however, that schizophrenia rarely presents before the age of 25 years ${ }^{14,17}$. None of the studies 1 , 2,15 attempted to quantify the strabismus in the study sample. Our study therefore investigated a sample including different races and a broader population between the ages of 15-70 years. Subjects over the age of 70 were excluded as people over this age are predisposed to developing a strabismus due to loss of muscle tonus with age ${ }^{26}$. The aim of the present study was to determine in Kwazulu Natal the prevalence of strabismus amongst a diverse population of schizophrenic subjects.

\section{Methods}

\section{Study design and sample}

A cross sectional study design was used. Ethical clearance was obtained from the Faculty of Health Sciences at the University of Kwazulu Natal prior to commencement of the fieldwork (FECHSC 028/10). The study population comprised schizophrenic subjects residing at the Durban and Coastal Mental Health (DMH) Institution and a saturated sample of 50 subjects was obtained. Consultation with a qualified psychologist helped to determine the inclusion and exclusion criteria. The inclusion criteria were 
subjects that had been diagnosed with schizophrenia, who were between the ages of 15 and 70 years and of either gender. Subjects that had any other mental or systemic illnesses and those who were on any medication other than that used to treat schizophrenia were excluded from the study. This information was obtained from the subjects' medical records. The manager of DMH granted permission for the usage of the premises and patients. They informed the subjects and determined possible participants according to the inclusion and exclusion criteria. Informed consent was obtained from the subjects and their legal care givers prior to commencement of the testing.

\section{Study procedure}

The battery of tests performed included observation, the cover test together with a prism bar and the modified Krimsky test. Subjects were observed for any abnormal head postures or face turns that may have been adopted to compensate for diplopia induced by the strabismus. The data gathering instruments used were an occluder to reveal the strabismus, a penlight torch to maintain fixation and view the corneal reflexes, and a prism bar to measure and realign the corneal reflexes relative to the midline of the pupil. To ensure the consistency of the testing instruments, one prism bar was used and the batteries of the penlight torch were changed as required to ensure consistent brightness. To ensure standardisation and to give a level of quality control each technique was performed by two researchers. Differences greater than two prism dioptres between the researchers were disregarded and the techniques repeated. This decision was made by a third researcher.

The cover test is the most common, efficient and reliable objective method to assess a strabismus ${ }^{2}$. It gives information on the magnitude, direction, laterality and stability of a deviation. In the present study it was performed with the relevant target, which was a letter one line above the unaided visual acuity of the poorer eye, at distance $(6 \mathrm{~m})$ and then at near $(40 \mathrm{~cm})$. The unilateral cover test was used to detect a strabismus by observation of the uncovered eye and the alternating cover test was used to measure the magnitude of the strabismus in conjunction with a prism bar using the appropriate base directions.

Following the cover test, the modified Krimsky test was performed. The fixation target was a penlight torch held in the midline at $50 \mathrm{~cm}$ from the subject and the examiner stood directly in front of the deviating eye to avoid errors of parallax ${ }^{27}$. The position of the corneal reflection of the light in each eye was observed by the examiner (DN/NY) while the other eye was covered. The examiner determined which eye was the deviating eye and placed appropriate prisms in front of this eye until normal alignment $(0.50 \mathrm{~mm}$ nasal from the centre of the pupil) was achieved. The base directions of the prisms were the same as in the alternating cover test depending on the direction of the deviation. The amount of prism to accomplish alignment was recorded as the magnitude of the strabismus. Von Noorden ${ }^{27}$ concluded that the advantages of the modified Krimsky test include independence from the fixation behaviour of the deviated eye, and an estimate of the deviation in subjects even with eccentric fixation.

Due to the nature of the subjects included in this study subjective methods to detect strabismus may not have been entirely reliable therefore both tests to investigate the strabismus were chosen based on their objectiveness and efficiency. Both tests were done unaided as this study was aimed at determining the prevalence of a habitual strabismus in the study sample. This however, is acknowledged as a limitation in this study as the strabismus could have been affected by poor visual acuity, refractive problems as well as accommodative anomalies hence the tests should have been done with the appropriate refractive corrections.

The methods used in this study to investigate the strabismus are similar to those used by previous studies $^{1,2}$. Toyota et al ${ }^{2}$ used similar procedures implementing both the unilateral and alternating cover test but using a Hirschberg test rather than a modified Krimsky test. The modified Krimsky test was chosen in the present study because it allows for the measurement of the deviation unlike with the Hirschberg test. Mohney et al $^{1}$ used the modified Krimsky test to identify and measure deviations in his study investigating the link between strabismus and schizophrenia. Hence, these tests are documented as being valid and reliable for parameters assessed in the present study.

Data was captured and analysed using the Statistical Package for Social Sciences (SPSS, version 15.0) under the guidance of the faculty statistician. The data collected was described in terms of frequencies and 
means, and the Pearson's chi-squared test was used to investigate correlations. Statistical significance was set at the $95 \%$ confidence level.

\section{Results}

\section{Demographics}

The 50 subjects were between fifteen and seventy year of age with a mean age of $50.45 \pm 11.12$ years. Of the sample $52 \%$ were female and $48 \%$ male showing an almost equal gender distribution. With regard to race, the most prevalent race in the sample were Indian (36\%) followed by Africans (30\%) and then Coloureds and Caucasians representing 24\% and $10 \%$ of the sample respectively. The race distribution of the sample is attributed to the location of DMH and not an indication of the prevalence of schizophrenia in different ethnic groups.

\section{Gender}

Of the subjects that presented with a strabismus, $48.6 \%$ were female as opposed to $51.4 \%$ males. The Pearson Chi Square test revealed that there was no gender bias in those subjects presenting with a strabismus $(p=0.424)$.

\section{Strabismus characteristics}

As illustrated by Figure 1, the prevalence of strabismus, detected by any one of the three methods used, and either at distance, near or both, was $74 \%$ $(n=37)$.

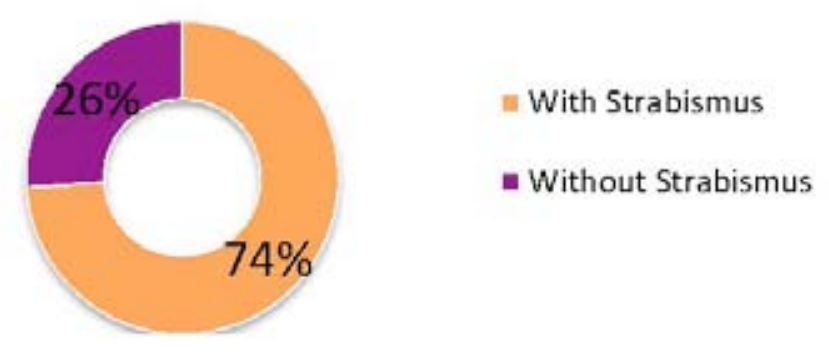

Figure 1: Prevalence of Strabismus

Irrespective of the test used, of the 37 subjects who presented with a strabismus, either at distance, near or both, $24 \%(n=9)$ had an esotropia and $76 \%(n=28)$ were exotropic (see Figure 2).

\section{$24.32 \%$}

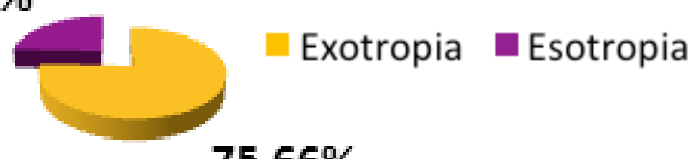

\section{$75.66 \%$}

Figure 2: Direction of Strabismus

Only one subject $(2.7 \%)$ presented with a vertical deviation (a hypertropia in addition to the esotropia). Furthermore, $13.5 \%(n=5)$ of the strabismic subjects presented with a deviation at both distance and near compared to $86.5 \%(n=32)$ of the strabismic subjects who only had a deviation when fixating at near as determined by either the near cover test or the modified Krimsky test.

Table 1 shows the prevalence of the strabismus with any one of the three tests. As can be seen in Table 1, the majority of the strabismus was detected with the patient fixating at near, and by the modified Krimsky test. There was no correlation found between the different methods used to detect and measure the strabismus in this study. The modified Krimsky test superseded the cover test both at distance and at near for the detection of the strabismus, with the modified Krimsky test detecting $86.5 \%$ of the strabismus that was present in the sample.

The mean, median, mode and range of the strabismus detected by the cover test at distance and at near and with the modified Krimsky test are shown in Table 2. With the subject fixating a distant target, even though only five subjects presented with a strabismus, the magnitude was quite significant with the average exotropia being 33 prism dioptres (pd). The average exotropia detected with the subject fixating a near target was lower being $11 \mathrm{pd}$ as detected with the near cover test and 9 pd with the modified Krimsky test. The average magnitude of the esotropia detected was less than that of the average exotropia being $6 \mathrm{pd}$ as detected with the distance cover test and $6 \mathrm{pd}$ as detected with the modified Krimsky test. The vertical deviation detected in only one of the subjects was a left hypertropia of $2 \mathrm{pd}$.

From the case history it was found out of the 74\% who had strabismus, only $16 \%$ were aware of the deviations and presented with obvious deviations upon being observed. It was of concern though that none of them had any intervention regarding the strabismus but unfortunately the reasons for this were not ascertained. 
Table 1: Prevalence of the strabismus detected, with the use of different tests in a sample of schizophrenic subjects

\begin{tabular}{|l|l|l|l|}
\hline & COVER TEST (@6 m) & $\begin{array}{l}\text { COVER TEST } \\
(@ 40 \mathrm{~cm})\end{array}$ & $\begin{array}{l}\text { MODIFIED KRIMSKY } \\
(@ 50 \mathrm{~cm})\end{array}$ \\
\hline Exotropia (XOT) & $8.11 \%(n=3 / 37)$ & $35.14 \%(n=13 / 37)$ & $62.16 \%(n=23 / 37)$ \\
\hline Esotropia (SOT) & $2.70 \%(n=1 / 37)$ & $0 \%(n=0 / 37)$ & $24.32 \%(n=9 / 23)$ \\
\hline Hypertropia & $2.70 \%(n=1 / 37)$ & $0 \%(n=0 / 37)$ & $0 \%(n=0 / 37)$ \\
\hline Hypotropia & $0 \%(n=0 / 37)$ & $0 \%(n=0 / 37)$ & $0 \%(n=0 / 37)$ \\
\hline Total & $13.51 \%(n=5 / 37)$ & $35.14 \%(n=13 / 37)$ & $86.49 \%(n=32 / 37)$ \\
\hline
\end{tabular}

Table 2: Magnitude of the strabismus detected in the sample using the three tests. All measurements are in prism dioptres (pd).

\begin{tabular}{|l|l|l|l|l|l|l|}
\hline & \multicolumn{3}{|l|}{$\begin{array}{l}\text { COVER TEST } \\
(@, 6 \mathrm{~m})\end{array}$} & \multicolumn{2}{l|}{$\begin{array}{l}\text { COVER TEST } \\
(@, 40 \mathrm{~cm})\end{array}$} & \multicolumn{2}{l|}{$\begin{array}{l}\text { MODIFIED KRIMSKY } \\
(@, 50 \mathrm{~cm})\end{array}$} \\
\hline & XOT & SOT & XOT & SOT & XOT & SOT \\
\hline MEAN & $33.33 \pm 20.21$ & $6 \pm 0$ & $10.62 \pm 7.5$ & 0 & $9.22 \pm 9.96$ & $6.22 \pm 1.86$ \\
\hline MEDIAN & 45 & 6 & 8 & 0 & 6 & 6 \\
\hline MODE & 45 & 6 & 8 & 0 & 4 & 6 \\
\hline RANGE & $10-45$ & - & $2-30$ & 0 & $4-40$ & $4-10$ \\
\hline
\end{tabular}

Table 3: Frequency and laterality of the strabismus detected using three tests in 50 schizophrenics based at DMH, Kwazulu-Natal, South Africa

\begin{tabular}{|c|c|c|c|c|c|c|c|}
\hline & & \multicolumn{2}{|c|}{$\begin{array}{l}\text { COVER } \\
\text { TEST }(@ 6 \mathrm{~m})\end{array}$} & \multicolumn{2}{|c|}{$\begin{array}{l}\text { COVER TEST } \\
((a, 40 \mathrm{~cm})\end{array}$} & \multicolumn{2}{|c|}{$\begin{array}{l}\text { MODIFIED KRIMSKY } \\
(@ 50 \mathrm{~cm})\end{array}$} \\
\hline & & XOT & SOT & $\mathrm{XOT}$ & SOT & $\mathrm{XOT}$ & SOT \\
\hline \multirow[t]{3}{*}{ Frequency } & Constant & $80 \%$ & 0 & $92 \%$ & 0 & $72 \%$ & $28 \%$ \\
\hline & Intermittent & 0 & $20 \%$ & $8 \%$ & 0 & 0 & 0 \\
\hline & undetermined & \multicolumn{2}{|c|}{$20 \%$ (L hypertropia) } & 0 & 0 & 0 & 0 \\
\hline \multirow[t]{3}{*}{ Laterality } & Unilateral & $40 \%$ & $20 \%$ & $62 \%$ & 0 & $47 \%$ & $25 \%$ \\
\hline & Alternating & $20 \%$ & 0 & $38 \%$ & 0 & $16 \%$ & $3 \%$ \\
\hline & undetermined & \multicolumn{2}{|c|}{$20 \%$ (L hypertropia) } & 0 & 0 & $9 \%$ & 0 \\
\hline
\end{tabular}

Table 3 above classifies the strabismus as detected by the three tests in terms of their frequency and laterality. With respect to frequency, the majority of the strabismus detected by any one of the three tests appeared to be constant exotropia. Furthermore, irrespective of the direction of the strabismus, in the majority of the subjects the strabismus appeared to be unilateral. However, it was not uncommon to find an alternating strabismus.

\section{Discussion}

The prevalence of strabismus in this sample of 50 schizophrenic subjects at the DMH in Kwazulu-Natal was found to be $74 \%$ highlighting the association between schizophrenia and strabismus. Toyota et $a l^{2}$ found a prevalence of strabismus of $13 \%$ in a sample of 346 schizophrenic subjects. Comparatively the prevalence of strabismus in the control group (5\%) in the study by Toyota et $a l^{2}$ was less than half that in the schizophrenic group indicating a higher prevalence amongst schizophrenic subjects. The difference in the prevalence established by this study and that of Toyota $e t a l^{2}$ could be related to the measurement techniques used which may have been influenced by subject co-operation and attention. Furthermore, the difference in sample size may have also resulted in varying results as the study by Toyota et $a l^{2}$ included 346 schizophrenic subjects compared to the 50 subjects examined in our study. The present study assessed the strabismus without any refractive correc- 
tion which could have affected the presentation of the strabismus. It is unclear as to whether or not the study by Toyota $e t a^{2}$ assessed the strabismus with the appropriate refractive correction used. Approximately $13 \%$ of subjects in our study presented with a distance strabismus when assessed with the cover test. This compares more closely to the findings of Toyota et $a l^{2}$. The study by Mohney et $a l^{1}$ found that $41.3 \%$ of subjects with a history of childhood strabismus also had a mental health disorder. Early on Hinton et al had speculated that mental disorders for example psychosis may arise due to the cosmetic influence of the strabismus. More recently, Toyota et al ${ }^{2}$ discovered that both schizophrenia and strabismus share a common defective gene. This gene, known as PMX2B, may be the reason that they present concurrently.

Speculation exists with respect to the side effects of drugs used to treat schizophrenia and their ocular effects. The most common medications used for schizophrenia were found to be haloperidol and phenerine based on the subjects' medical records. It has been found that schizophrenia medication may present with induced intraocular lens and corneal pigmentary changes which may produce visual impairment such as halos around lights, hazy vision, photophobia, and watering eyes ${ }^{28}$. Other ocular side effects include increased blinking, inability to move the eyes, miosis or mydriasis with loss of accommodation, narrow angle glaucoma, esophoria, impaired fusional convergence and divergence amplitudes ${ }^{28}$. Not all individuals experience some or all of the side effects as side effects are found to be dependant on the duration of the therapy and dosage of each medication with the side effects disappearing with withdrawal of the medication. Furthermore, neuroleptics such as haloperidol that are used in the treatment of schizophrenia can result in a locking of the eyes in an elevated position which is very different from that of a strabismus ${ }^{2}$.

The majority of the subjects with strabismus in the present study were found to be exotropic (76\%) compared to only $24 \%$ with an esotropia. Toyota et al found strabismus in $13 \%$ of schizophrenic subjects with $95 \%$ of these subjects being exotropic and only one subject (2.17\%) being esotropic. Mohney et al ${ }^{1}$ did a retrospective study on the prevalence as well as the types of psychiatric disorders that were diagnosed by adulthood in a sample of 266 esotropic subjects and 141 exotropic subjects who had strabismus as children and who were residing at Olmsted County, Minnesota between January 1, 1985 and December 31, 1994. Forty five percent of the esotropic subjects were female, and $58.2 \%$ of the exotropic subjects were female. The racial distribution was $95.7 \%$ White, 3\% Asian American, 0.7\% Black and 0.3\% each of Native American and other. Exotropic children appeared to have a three times ( $95 \%$ confidence interval: 1.9-5.1 times) greater likelihood of developing a mental illness compared to orthotropic children in the control group ${ }^{1}$, and yet esotropic children did not appear at any greater risk of developing a psychiatric disorder when compared to the orthotropic children ${ }^{1}$. Toyota et $a l^{2}$ also reported an odds ratio for constant exotropia in schizophrenia was $20.6 \%$ (95\% confidence interval, 5.03-56.2).

The genes that have been linked to the association between strabismus and schizophrenia are also responsible for the development of the third and fourth cranial nerves ${ }^{25}$. These nerves innervate, amongst other muscles, the medial rectus. The medial rectus is primarily involved in adduction of the eye; hence defective innervations are expected to result in an exotropia. The lateral rectus muscle is innervated by the sixth cranial which is not controlled by these genes ${ }^{25}$. If the lateral rectus was affected it would result in an esotropia. Furthermore, in comparison to esotropia, exotropia has a more pronounced adverse cosmetic appearance and is easier to be detected even by a lay person just by observation and may therefore have a greater negative impact on a person's self image ${ }^{12,29,30}$. The prevalence of a vertical deviation in both the present study as well as that by Toyota et $a l^{2}$ was very low, being $2.7 \%$ and $2.17 \%$ respectively, which may indicate that the vertical position of the eye and the muscles responsible are not as affected by defects in the PMX2B and ARIX genes.

In our study, the majority of the strabismus ( $86.5 \%)$ was diagnosed with the subject fixating a near target. When one fixates a near target convergence is required which once again calls on the actions of the medial rectii muscles. Deficiencies in convergence can thus result in the manifestation of an exotropia. The study by Mohney et a ${ }^{1}$ did record a greater prevalence of convergence insufficiency in children with mental disorders such as schizophrenia, psychoses and bipolar disorder. Most of the strabismus detected in this study was with the use of the modified Krimsky test. There was no significant correlation found 
between the different tests used to detect and measure the strabismus in this study. Possible reasons for this discrepancy between the techniques are that the deviations may not have been detected with the cover test if there was eccentric fixation present especially if the angle of eccentric fixation was equal to the magnitude of the strabismus. Future studies should take eccentric fixation into account when determining the presence or absence of a strabismus. The other explanation for the differences between the techniques was the fixation behavior of the subjects, who tended to be wary of the presence of the occluder during the cover test, thus contaminating results of this procedure to an extent. In the modified Krimsky test, the prism to reposition the corneal reflex is placed over the fixating eye, that is, the secondary angle of strabismus is measured, however, with the alternating cover test the prism is placed over the non-fixating eye and hence the primary angle is being measured. In order for the modified Krimsky test to be accurately interpreted however, extraocular motilities must be full ${ }^{31}$. This was not checked as part of this study and should be included in future studies investigating strabismus in schizophrenia. This study was done by final year optometry students, and minimal experience with the testing procedures and exposure to the subject population may also have contributed to discrepancies in the findings.

A difference in the magnitude of the strabismus when fixating at distance compared to near, as well as, with exotropia compared to esotropia was noted. The mean exotropia at distance was $33 \mathrm{pd}$ and the means when fixating at near were 11 pd with the near cover test and 9 pd with the modified Krimsky test. Previous studies ${ }^{1,5}$ have not reported on the actual magnitude of the strabismus in schizophrenic patients. Statistics around the magnitude of the strabismus are vital when one considers the implication that a noticeable strabismus has an effect on the psychosocial functioning of an individual. The cosmetic appearance of a noticeable heterotropia can affect a patient emotionally $^{4}$. Larson et al ${ }^{29}$ found that the threshold for the detection of a horizontal heterotropia was found to be 12.5 prism dioptres. Weissberg et $a^{30}$ reported that an angle of 14.5 prism dioptres for esotropes and 8 prism dioptres for exotropes was noticeably visible by lay observers. Even though the mean strabismus when fixating a near target reported in the present study are lower than the threshold values suggested above, it is important to note that ranges illustrated in Table 2. The upper limit of the ranges was much larger than the threshold values found by previous studies. Thus, the additional benefit of squint surgery on the psychosocial functioning of a person is becoming more apparent in recent times ${ }^{13}$.

With respect to the frequency, the majority of the strabismus was found to be constant (Table 3). Similar findings were reported by Toyota et $a l^{2}$. The exact reason for why constant exotropia predominates in schizophrenia however remains unknown ${ }^{2}$. Mohney et al ${ }^{1}$ also reported that subjects with a constant exotropia appeared to be prone to developing a psychiatric disorder earlier than in those with an intermittent exotropia. Even though some of the subjects in the present study presented with an alternating strabismus majority were unilateral (Table 3 ).

Debate does exist among recent studies as to whether there is a gender bias with mental illness. Mohney et al ${ }^{1}$ revealed no gender difference with respect to the prevalence of mental illness. McKenzie et $a l^{32}$ on the other hand, found mental illness to be more prevalent in males than females in subjects that had intermittent exotropia. There did not appear to be a gender bias with respect to the prevalence of strabismus among the schizophrenic subjects in the present study.

\section{Conclusion}

This study showed a high prevalence of constant exotropia in schizophrenic patients. Among individuals who later develop schizophrenia in adulthood there have been limited studies investigating extraocular muscle dysfunction in childhood and this study thus adds to that knowledge base. This study was not without limitations as it was a cross-sectional study done on a limited number of subjects. Furthermore, the strabismus was assessed without the refractive compensation which could have altered the presentation. Future studies into this area are important and will help address the issue of whether extraocular muscle disorders are a function of schizophrenia or a precursor to the disorder. It would benefit healthcare professionals in the management strategies when dealing with paediatric patients with strabismus and/ or disorders such as schizophrenia. 


\section{Acknowledgements}

We acknowledge the support and guidance of Miss M Manillal and Dr B Mohney in the course of the study.

\section{References}

1. Mohney BG, McKenzie JA, Capo JA, Nusz KJ, Mrazek D, Diehl NN. Mental illness in young adults who had strabismus as children. Pediatrics 2008 122(5)1033-1038.

2. Toyota T, Yoshitsugu K, Ebihara M, Yamada K, Ohba H, Fukasawa M, Minabe Y, Nakamura K, Sekine Y, Takei N, Suzuki K, Itokawa M, Meerabux JMA, Iwayama-Shigeno Y, Tomaru Y, Shimizu H, Hattori E, Mori N, Yoshikawa T. Association between schizophrenia with ocular misalignment and polyalanine length variation in PMX2B. Human Molecular Genetics 200413 (5) 551-56.

3. Dale RT. Fundamentals of Ocular Motility and Strabismus. University of Michigan: Grune \& Stratton, 1982.

4. Eskridge JB, Amos JF, Bartlett JD. Clinical Procedures in Optometry. Philadelphia: Lippincott, 1991.

5. Bankes JLK. Clinical Ophthalmolology. New York: Churchill Livingstone, 1987.

6. Coakes R, Sellors PH. Outline of Ophthalmology. Oxford: Butterworth Heinemann Ltd, 1995.

7. Akay AP, Cakaloz B, Berk T, Pasa E. Psychosocial aspects of mothers of children with strabismus. Journal of American Association for Pediatric Ophthalmology and Strabismus 20059 (3) 268-273.

8. Hinton GG. Childhood psychosis or mental retardation: A diagnostic dilemma. Canadian Medical Association Journal 196389 1020-1024.

9. Satterfield D, Keltner JL, Morrison TL. Psychosocial aspects of strabismus study. Archives of Ophthalmology 1993 111(8) 1100-1105.

10. Olitsky SE, Sudesh S, Graziano A, Hamblen J, Brooks SE, Shaha SH. The negative psychosocial impact of strabismus in adults. Journal of American Association for Pediatric Ophthalmology and Strabismus 1999 3(4) 209-211.

11. Sabri K, Knapp CM, Thompson JR, Gottlob I. The VF-14 and psychological impact of amblyopia and strabismus. Investigative Ophthalmology and Vision Science 2006 47(10) 4386-4392.

12. Tonge BJ, Lipton GL, Crawford G. Psychological and educational correlates of strabismus in school children. Australian and New Zealand Journal of Psychiatry 198418 71-77.

13. Durnian JM, Noonan CP, Marsh IB. The psychosocial effects of adult strabismus - a review. British Journal of Ophthalmology 201195 (4) 450.

14. Lewis DA, Lieberman JA. Catching up on schizophrenia: Natural history and neurobiology. Neuron 200026 325-334.

15. Schiffman J, Maeda JA, Hayashi K, Michelsen N, Sorensen HJ, Ekstrom M, Abe KA, Chronicle EP, Mednick SA. Premorbid childhood ocular alignment abnormalities and adult schizophrenia-spectrum disorder. Schizophrenia Research 200681 253-260.
16. Tsuang MT, Faraone SV, Johnson PDC. Schizophrenia: The Facts. Oxford: Oxford University Press, 1997.

17. Raulin ML. Abnormal Psychology, United States of America: Pearson Education Inc, 2003.

18. Davidson GC. Abnormal Psychology. New York: John Wiley, 2004.

19. Sharma T, Harvey PD. The Early Course of Schizophrenia. Oxford: Oxford University Press, 2006.

20. Shives LR. Basic Concepts of Psychiatric-Mental Health Nursing. Philadelphia: Wolters Kluwer/ Lippincott Williams \& Wilkins, 2008.

21. Gottesman IL. Schizophrenia Genesis: The Origins of Madness. New York: Library of Congress Cataloging-in-publication Data, 1991.

22. Kathmann N, Hochrein A, Uwer R and Bondy B. Deficits in gain of smooth pursuit eye movements in schizophrenia and affective disorder patients and their unaffected relatives. American Journal of Psychiatry 2003160 696-702.

23. Ettinger U, Kumari V, Crawford TJ, Corr PJ, Das M, Zachariah E, Hughes C, Sumich AL, Rabe-Hesketh S, Sharma T. Smooth pursuit and antisaccade eye movements in siblings discordant for schizophrenia. Journal of Psychiatric Research 200438 177-184.

24. Nkam I, Bocca M, Denise P, Paoletti X, Dollfus S, Levillain $\mathrm{D}$, Thibaut F. Impaired smooth pursuit in schizophrenia results from prediction impairment only. Biological Psychiatry 200967 992-997.

25. Nakano M, Yamada K, Fain J, Sener EC, Selleck CJ, Awad AH, Zwaan J, Mullaney PB, Bosley TM, Engle EC. Homozygous mutations in ARIX (PHOX2A) result in congenital fibrosis of the extraocular muscles type 2. Nature Genetics 293 315-20.

26. Clark RA, Demer JL. Effect of aging on human rectus extraocular muscle paths demonstrated by magnetic resonance imaging. American Journal of Ophthalmology 2002346 872-878.

27. Von Noorden GK. Binocular Vision and Ocular Motility. London: CV Mosby Company, 1980: 192.

28. Hansen TE, Casey DE, Hoffman WF. Neuroleptic intolerance. Schizophrenia Bulletin 1997234 567-582.

29. Larson SA, Keech RV, Verdick RE. The threshold for the detection of strabismus. Journal of American Association for Pediatric Ophthalmology and Strabismus 200376 418422.

30. Weissberg E, Suckow M, Thorn F. Minimum angle horizontal strabismus detectable by lay observers. Optometry and Vision Science 2004817 505-509.

31. Kazlas M. Diagnosis and management of amblyopia. Ophthalmology Rounds 2007110 www.ophthalmologyrounds. org accessed 17 June 2011.

32. McKenzie JA, Capo JA, Nusz KJ, Diehl NN, Mohney BG. Prevalence and sex differences of psychiatric disorders in young adults who had intermittent exotropia as children. Archives of Ophthalmology 20091276 743-747. 